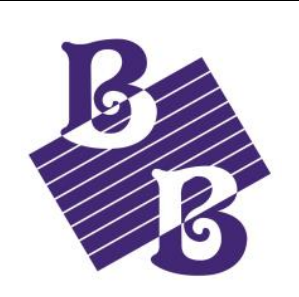

BioBacta
Journal of Bioscience and Applied Research

www.jbaar.org

\title{
Assessment of Safe Handling and Disposal of Laundry in Hemodialysis Unit Soheir Abouelfadle ${ }^{1}$, Thoraya Mohamed Abdel Aziz ${ }^{1}$, and Doaa Amin Ahmed Sayed Ahmed ${ }^{2}$ \\ ${ }^{1}$ Medical-Surgical Nursing Department, Faculty of Nursing, Alexandria University, Egypt \\ ${ }^{2}$ Medical-Surgical Nursing Department, Faculty of Nursing, Damanhour University, Egypt \\ *Corresponding author: Dr/ Thoraya Mohamed Abdelaziz Email:thoraied@yahoo.com
}

DOI: $10.21608 /$ JBAAR.2021.131143

\begin{abstract}
Handling laundry is a major task that must be managed safely by health care workers (HCW) for maintaining a green hospital environment. Laundry processing, especially in hemodialysis units is a source of many hazards, especially bacteriological ones. This study aimed to assess safe laundry handling and disposing in hemodialysis units. A descriptive research design was utilized. Subject A convenience sample of 46 nurses (nursing and auxiliary members) was enrolled in this study. Materials and method: The current study was conducted in dialysis units at the following Hospitals; Students' University and Health Insurance (Gamal Abd-El-Naser); Alexandria. Tool: One tool was utilized "Safety practices of laundry handling and disposing of in hemodialysis units: Observational Checklist"; it was developed by the researchers after a thorough review of related literature. It comprised two parts, Part I: Personal data such as age, gender, years of experience, employment position, educational level, and previous attendance in-service training regarding safe laundry management. Part II: Safety Laundry Handling and Disposing Observational Checklist. Result: This study reflected satisfactory safe practices level of laundry handling scores in both settings. While, no significant correlation was detected between both settings regarding safe practices of laundry handling and disposal, a significant relationship between overall practice levels and staff sociodemographic characteristics except gender was noticed. Conclusion: A significant correlation between the availability of all-time PPE and laundry processing; with satisfactory scores for applying safety laundry practices was noticed. Recommendations: Periodic monitoring for staff as well as continuous training programs regarding safe laundry processing must take place. All linen must be handled, stored, and transported in a manner that maintains a green environment for all patients, health care workers, as well as visitors for the sustainability of health and safety in hemodialysis units.
\end{abstract}

Keywords: Safe, Laundry processing, Green hospital environment, Health caregivers (HCGs), and Hemodialysis. 


\section{Introduction}

Maintaining a green environment is a fundamental requirement for all, by providing safe health care practices; in general. However, one of the most critical areas; is dealing with laundry as a very serious task through storage, handling, bagging, and transportation. In spite, of considering laundering as a source of health care setting hazard; there is no clear direct attention to this issue ${ }^{(1)}$. Although acquiring infection from the laundry is a low risk even if contaminated with blood and body fluid (CDC2000); it is seriously motivated that all laundry must be adequately washed, dried, and correctly stored. ${ }^{(2)}$

Dialysis is one of the renal replacement therapy that can cause numerous hazards. Nevertheless, the hemodialysis unit is a high source area of contamination with blood and body fluid. However, infection risk transmission can be reduced thru restrict infection control (IC) practices (3). Accordingly, hemodialysis health caregivers (HCGs) have a major responsibility to assure that, patients and colleagues have not been exposed to the hemodialysis expected hazards, by maintaining safe practices of care ${ }^{(1)}$. Thus, it is urgent to set and apply policy as well as a standard for laundry processing; highlighting its cautious handling and transfer' for minimizing exposure to hospitals' infection risk ${ }^{(1,3)}$.

Therefore, policies had to declare that; clean linen must be delivered in covered cages and stored in a specialized designated area for protection from dirt, dust, or water contamination e.g. kept in a cupboard which must be away from the dirty floor, bathrooms or trolleys. Also, emphasize IC universal precautions, especially for staff processing laundry ${ }^{(4,5,6)}$. Otherwise, emphasis should be directed on the safe handling of dirty or soiled laundry from human secretions and that containing unnoticeable sharp objects, protect patients, HCGs, and visitors from environmental aerosolizing bacteria and additional mechanical hazards. ${ }^{(7)}$
Nevertheless, personal protective equipment (PPE) such as gloves, eye goggles, and aprons must be worn when handling dirty soiled laundry to prevent staff and their uniform from being contaminated $^{(8)}$. Otherwise, the used laundry must be placed into colored coding bags with less than two-thirds fullness and securely closed to prevent the likelihood of dropping or splitting. Furthermore, dirty laundry must be placed in white plastic bags, while soiled must be sealed in red plastic bags; patients' laundry must be sent to their homes in a plastic bag. ${ }^{(7,8)}$

Hence, safe laundry processing can be achieved through HCGs and IC team cooperation; to ensure staff guidance, access, and compliance with the safe laundry processing policy. In addition to safeguarding the availability and adequacy of PPE, besides increasing HCGs consensus for checking the presence of sharp objects in the laundry before processing. Along with designing and implementing a training plan about safe and correct handling and transfer of laundry; to control infection risk or other laundry dealing hazards as possible $^{(2,9)}$.

\section{Study significance:}

The present study will afford a backbone not just for laundry processing analysis for the academic or scientific community, but also will provide practical benefits regarding informing the staff of all needed policies and training plans for helping the health care agencies to save money and effort. As well, as to place a flashlight on negligible handling, transporting, and laundering processes. Furthermore, help to signify alarms about safety and its effect on health sustainability, and enhance the warrens of green for health care setting environment in general.

Aim of the study: To assess the safe handling and disposal practices of laundry in the hemodialysis unit.

\section{Research questions:}

Is there safe laundry handling and disposing of in the hemodialysis unit? 
What is the safety of laundry handling and disposing of in hemodialysis units?

\section{Materials and method}

Materials

Study design: It is a descriptive research design.

Setting: The study was carried out at two hemodialysis units in Alexandria - Egypt: namely; Students' University Hospital and Health Insurance Hospital (Gamal Abd-El-Naser).

Sample: A convenient sample of all working staff $\mathrm{N}=46$ (nurses and nurses auxiliary) at the mentioned settings were available during data collection time and responsible for laundry processing. (Students' University Hospital $\mathrm{N}=22$, Health Insurance Hospital N=24).

Tool: One tool was utilized: "Safety laundry handling and disposal in Hemodialysis units: Observational Checklist", it was developed by researchers after a thorough review of related literature ${ }^{(8,10,11)}$. It aimed to assess safety processing as handling and disposal of laundry in the hemodialysis unit. It comprised two parts: Part I: "Laundry processing as handling and staff sociodemographic data" as; age, gender, years of experience, position, education, and previous training regarding laundry processing. Part II: "Safety Laundry processing as handling and disposal assessment checklist", aimed to assess the assigned staff laundry processing as handling and disposal practices in the hemodialysis unit. It consisted of 5 safe laundry processing practices; Clean laundry disposal (7 items), Soiled/contaminated laundry disposal, (7 items), isolated or infected laundry disposal, comprised (4 items), laundry handling staff; infection control practices (6 items) and laundry processing in general, (6 items).

Scoring system: Laundry processing practices were scored as Yes $=2$, Somehow $=1, \mathrm{No}=0$. Each practice included sub-items; which were scored by 3 points Likert scale: Done correctly $=2$, incorrectly done $=1$, Not done $=0$. Analyzing safe laundry handling designs was under two main categories: equal or above $60 \%$ was considered satisfactory and less than $60 \%$ was unsatisfactory. Checklist total scoring was performed by adding items on each of the subscales.

\section{Method}

1- Permission to carry out the current study was attained from hemodialysis unit heads after an explanation of the study's purpose.

2- Tool developed by the researchers after reviewing the related literature, ${ }^{(8,10,11)}$ and revised by the jury of 5 experts in a related field, to test its validity, and all necessary modifications were done accordingly. Cronbach- alpha coefficient test was utilized for reliability, results illustrate that the tool reliability $=0,726$.

3- A pilot study was conducted on seven staff for test the tool's clarity, feasibility, and applicability, and necessary modifications were done.

4- Data Collection: The observational checklist was filled by researchers through indirect intermittent observation of nursing personnel during laundry handling and disposal in each hemodialysis unit. They were assessed 3 hours/shift, in different shifts and all days/week except Friday (units' weekend), for assessing their laundry handling and disposing of practices. The average time for tool completion ranged from 30- 40 minutes for each staff member.

5- Data collection consumed 4 months from September to December 2018.

\section{6- Ethical considerations}

a. Written approval was obtained from the ethical committee of- Alexandria University Faculty of Nursing.

b. Informed consent was attained from participants after an explanation of the study's purpose and their right to withdraw from the study at any time was confirmed.

c. Information was managed anonymously, also privacy \& confidentiality was assured.

7- Statistical analysis: was done using SPSS version 20. Statistical analysis was done by utilizing twotailed tests \& a P value less than or equal to $\mathbf{0 . 0 5}$ was considered statistically significant.

\section{Results}


Table 1 shows, the subjects' characteristics homogeneity in both settings that; $95 \%$ were female, and more than $1 / 3$ of their age ranged between $30-39$ years, also their experience ranged from 10 to $\geq 20$ years (nearly $37 \%$ for each setting). Regarding position it was noticed that; nearby $50 \%$ were junior (newly graduated) in health insurance and students' University Hospital representing (45.5\%, \& 41.7\%) respectively, while seniors were $(22.7 \%$ \& $20.8 \%)$ respectively. Furthermore, educational level reflected that around $2 / 3$ had diploma level $(59.1 \%$, \& $58.3 \%)$ respectively and bachelor were $(27.3 \% \& 29.2 \%)$ respectively. Otherwise, the vast majority (87\%) in general didn't attend any training or workshops related to laundry processing. Furthermore, all subjects' reported that nurses and nursing aides are the laundry handler and responsible staff.

Table (1): Nurses' distribution according to socio-demographic characteristics $(n=46)$

\begin{tabular}{|c|c|c|c|c|c|c|c|}
\hline \multirow{3}{*}{ Variables } & \multirow{3}{*}{\multicolumn{2}{|c|}{$\begin{array}{l}\begin{array}{l}\text { Total } \\
(\mathrm{n}=46)\end{array} \\
\text { No. } \%\end{array}$}} & \multicolumn{4}{|c|}{ Hospital } & \multirow{3}{*}{$P$} \\
\hline & & & \multirow{2}{*}{\multicolumn{2}{|c|}{\begin{tabular}{|c|}
$\begin{array}{c}\text { A } \\
(\mathbf{n}=\mathbf{2 2})\end{array}$ \\
No. \\
$\%$ \\
\end{tabular}}} & \multirow{2}{*}{\multicolumn{2}{|c|}{\begin{tabular}{|l|}
\multicolumn{1}{|c|}{ B } \\
$(\mathrm{n}=24)$ \\
No. \\
$\%$
\end{tabular}}} & \\
\hline & & & & & & & \\
\hline $\begin{array}{c}\text { Gender } \\
\text { Male } \\
\text { Female }\end{array}$ & $\begin{array}{c}2 \\
44 \\
\end{array}$ & $\begin{array}{r}4.3 \\
95.7 \\
\end{array}$ & $\begin{array}{c}1 \\
21 \\
\end{array}$ & \begin{tabular}{|c|}
4.5 \\
95.5
\end{tabular} & $\begin{array}{c}1 \\
23 \\
\end{array}$ & $\begin{array}{c}4.2 \\
95.8\end{array}$ & $\begin{array}{c}{ }^{{ }^{F E}} p= \\
1.000\end{array}$ \\
\hline \begin{tabular}{|l|} 
Age \\
$20-29$ \\
$30-39$ \\
$40-49$ \\
$50-59$ years \\
\end{tabular} & $\begin{array}{c}10 \\
17 \\
12 \\
7\end{array}$ & $\begin{array}{l}21.7 \\
37.0 \\
26.1 \\
15.2\end{array}$ & $\begin{array}{c}6 \\
10 \\
3 \\
3\end{array}$ & $\begin{array}{l}27.3 \\
45.5 \\
13.6 \\
13.6\end{array}$ & $\begin{array}{c}4 \\
10 \\
7 \\
3\end{array}$ & $\begin{array}{l}16.7 \\
41.7 \\
29.2 \\
12.5\end{array}$ & $\begin{array}{r}{ }^{\mathrm{MC}} p= \\
0.621\end{array}$ \\
\hline $\begin{array}{l}\text { Years of experience } \\
\geq 10 \text { years } \\
10 \geq 20 \text { years } \\
20 \geq 30 \text { years } \\
\text { More than } 30 \text { years }\end{array}$ & $\begin{array}{c}10 \\
17 \\
12 \\
7\end{array}$ & $\begin{array}{l}21.7 \\
37.0 \\
26.1 \\
15.2\end{array}$ & $\begin{array}{l}6 \\
8 \\
5 \\
3\end{array}$ & \begin{tabular}{l|}
27.3 \\
36.4 \\
22.7 \\
13.6
\end{tabular} & $\begin{array}{l}4 \\
9 \\
7 \\
4\end{array}$ & $\begin{array}{l}16.7 \\
37.5 \\
29.2 \\
16.7\end{array}$ & $\begin{array}{l}{ }^{\mathrm{MC}} p= \\
0.871\end{array}$ \\
\hline \begin{tabular}{|l|} 
Position \\
Nursing aides staff \\
Junior staff (newly \\
graduate) \\
Senior staff (diploma) \\
Supervisor nurse \\
Head-nurse \\
\end{tabular} & $\begin{array}{c}6 \\
20 \\
\\
10 \\
8 \\
2\end{array}$ & \begin{tabular}{c|}
13.0 \\
43.5 \\
\\
21.7 \\
17.4 \\
4.3 \\
\end{tabular} & $\begin{array}{c}3 \\
10 \\
\\
5 \\
3 \\
1\end{array}$ & \begin{tabular}{|c|}
13.6 \\
45.5 \\
\\
22.7 \\
13.6 \\
4.5 \\
\end{tabular} & $\begin{array}{c}3 \\
10 \\
\\
5 \\
5 \\
1\end{array}$ & \begin{tabular}{c|}
12.5 \\
41.7 \\
\\
20.8 \\
20.8 \\
4.2 \\
\end{tabular} & $\begin{array}{l}{ }^{\mathrm{MC}} p= \\
0.968\end{array}$ \\
\hline $\begin{array}{l}\text { Education level } \\
\text { Diploma } \\
\text { Bachelor degree } \\
\text { Others }\end{array}$ & $\begin{array}{c}27 \\
13 \\
6\end{array}$ & $\begin{array}{l}58.7 \\
28.3 \\
13.0\end{array}$ & $\begin{array}{c}13 \\
6 \\
3\end{array}$ & $\begin{array}{l}59.1 \\
27.3 \\
13.6\end{array}$ & $\begin{array}{c}14 \\
7 \\
3\end{array}$ & $\begin{array}{l}58.3 \\
29.2 \\
12.5\end{array}$ & $\begin{array}{l}{ }^{{ }^{\mathrm{C}}} p= \\
1.000\end{array}$ \\
\hline \begin{tabular}{|l} 
Workshop attendance \\
Yes \\
No \\
\end{tabular} & $\begin{array}{c}6 \\
40 \\
\end{array}$ & $\begin{array}{l}13.0 \\
87.0\end{array}$ & $\begin{array}{c}2 \\
20 \\
\end{array}$ & $\begin{array}{c}9.1 \\
90.9\end{array}$ & $\begin{array}{c}4 \\
20 \\
\end{array}$ & $\begin{array}{l}16.7 \\
83.3\end{array}$ & $\begin{array}{c}{ }^{\mathrm{FE}} p= \\
0.667\end{array}$ \\
\hline $\begin{array}{l}\text { Laundry handling staff: } \\
\text { Both nurses \& nursing } \\
\text { aids }\end{array}$ & 46 & 100.0 & 22 & 100.0 & 24 & 100.0 & - \\
\hline
\end{tabular}


Table 2: demonstrates that most of the items related to clean laundry handling were done correctly by all nurses in both groups. On the other hand, two practices namely; "Linens are counted, sort then picked up"; and "Inspection to determine if intact" were done incorrectly by more than half of the subjects in both settings. However, there is no statistically significant difference was detected between both settings.

Table (2): Subjects distribution according to clean laundry handling ( $n=46)$

\begin{tabular}{|c|c|c|c|c|c|c|c|c|}
\hline \multirow{3}{*}{ Clean laundry handling } & \multirow{3}{*}{ Hospital } & \multicolumn{6}{|c|}{$\underline{\text { Done }}$} & \multirow{3}{*}{$\boldsymbol{P}$} \\
\hline & & \multicolumn{2}{|c|}{ correctly } & \multicolumn{2}{|c|}{ incorrectly } & \multicolumn{2}{|c|}{ Not done } & \\
\hline & & No. & $\%$ & No. & $\%$ & No. & $\%$ & \\
\hline 1. Linens are counted, sorted then picked up & $\begin{array}{c}\text { A } \\
\text { B } \\
\text { Total } \\
\end{array}$ & $\begin{array}{c}7 \\
12 \\
19 \\
\end{array}$ & $\begin{array}{l}31.8 \\
50.0 \\
41.3\end{array}$ & $\begin{array}{l}15 \\
12 \\
27 \\
\end{array}$ & $\begin{array}{l}\mathbf{6 8 . 2} \\
\mathbf{5 0 . 0} \\
\mathbf{5 8 . 7}\end{array}$ & $\begin{array}{l}\mathbf{0} \\
\mathbf{0} \\
\mathbf{0}\end{array}$ & $\begin{array}{l}0.0 \\
0.0 \\
0.0\end{array}$ & 0.211 \\
\hline 2. Linens are packed neatly in a plastic bag separately. & $\begin{array}{c}\text { A } \\
\text { B } \\
\text { Total }\end{array}$ & $\begin{array}{l}22 \\
24 \\
46\end{array}$ & $\begin{array}{l}100.0 \\
100.0 \\
100.0\end{array}$ & $\begin{array}{l}\mathbf{0} \\
\mathbf{0} \\
\mathbf{0}\end{array}$ & $\begin{array}{l}0.0 \\
0.0 \\
0.0\end{array}$ & $\begin{array}{l}\mathbf{0} \\
\mathbf{0} \\
\mathbf{0}\end{array}$ & $\begin{array}{l}0.0 \\
0.0 \\
0.0\end{array}$ & \\
\hline 3. Linens are inspected to determine if all were intact. & $\begin{array}{c}\text { A } \\
\text { B } \\
\text { Total }\end{array}$ & $\begin{array}{c}5 \\
11 \\
16\end{array}$ & $\begin{array}{l}22.7 \\
45.8 \\
34.8\end{array}$ & $\begin{array}{l}17 \\
13 \\
30 \\
\end{array}$ & $\begin{array}{l}77.3 \\
54.2 \\
65.2\end{array}$ & $\begin{array}{l}\mathbf{0} \\
\mathbf{0} \\
\mathbf{0}\end{array}$ & $\begin{array}{l}0.0 \\
0.0 \\
0.0\end{array}$ & 0.100 \\
\hline 4. Linens are inspected for cleanliness & $\begin{array}{c}\text { A } \\
\text { B } \\
\text { Total }\end{array}$ & $\begin{array}{l}22 \\
24 \\
46 \\
\end{array}$ & $\begin{array}{l}100.0 \\
100.0 \\
100.0\end{array}$ & $\begin{array}{l}\mathbf{0} \\
\mathbf{0} \\
\mathbf{0}\end{array}$ & $\begin{array}{l}0.0 \\
0.0 \\
0.0\end{array}$ & $\begin{array}{l}\mathbf{0} \\
\mathbf{0} \\
\mathbf{0}\end{array}$ & $\begin{array}{l}0.0 \\
0.0 \\
0.0\end{array}$ & - \\
\hline $\begin{array}{l}\text { 5. dirty linens collected \& returned to laundry } \\
\text { properly }\end{array}$ & $\begin{array}{c}\text { A } \\
\text { B } \\
\text { Total }\end{array}$ & $\begin{array}{l}21 \\
21 \\
42\end{array}$ & $\begin{array}{l}95.5 \\
87.5 \\
91.3\end{array}$ & $\begin{array}{l}\mathbf{0} \\
\mathbf{0} \\
\mathbf{0}\end{array}$ & $\begin{array}{l}\text { 0.0 } \\
0.0 \\
\text { 0.0 }\end{array}$ & $\begin{array}{l}1 \\
3 \\
4\end{array}$ & $\begin{array}{c}4.5 \\
12.5 \\
8.7\end{array}$ & $\begin{array}{c}{ }^{F E} p= \\
0.609\end{array}$ \\
\hline 6. Linens are placed in a special cabinet for storage & $\begin{array}{c}\mathbf{A} \\
\mathbf{B} \\
\text { Total } \\
\end{array}$ & $\begin{array}{l}22 \\
24 \\
46\end{array}$ & $\begin{array}{l}100.0 \\
100.0 \\
100.0\end{array}$ & $\begin{array}{l}\mathbf{0} \\
\mathbf{0} \\
\mathbf{0}\end{array}$ & $\begin{array}{l}0.0 \\
0.0 \\
0.0\end{array}$ & $\begin{array}{l}\mathbf{0} \\
\mathbf{0} \\
\mathbf{0}\end{array}$ & $\begin{array}{l}0.0 \\
0.0 \\
0.0\end{array}$ & - \\
\hline \begin{tabular}{|l} 
7. Laundry cycles are used according to the \\
manufacturer's recommendations.
\end{tabular} & $\begin{array}{c}\mathbf{A} \\
\mathbf{B} \\
\text { Total } \\
\end{array}$ & $\begin{array}{l}22 \\
24 \\
46\end{array}$ & $\begin{array}{l}100.0 \\
100.0 \\
100.0\end{array}$ & $\begin{array}{l}\mathbf{0} \\
\mathbf{0} \\
\mathbf{0}\end{array}$ & $\begin{array}{l}0.0 \\
0.0 \\
0.0\end{array}$ & $\begin{array}{l}\mathbf{0} \\
\mathbf{0} \\
\mathbf{0}\end{array}$ & $\begin{array}{l}0.0 \\
0.0 \\
0.0\end{array}$ & - \\
\hline
\end{tabular}

MC: Monte Carlo for Chi-square test

FE: Fisher Exact for Chi-square test

As noticed in table 3; $100 \%, 93.5 \%, 73.9 \%$, and $69,6 \%$, performed 5 out of 7 practices items correctly concerning "Soiled linen not sorted or rinsed in used location, Contaminated laundry placed\& transported in red bags or containers labeled as a biohazard, soiled linen inspected for any diapers, Place wet contaminated laundry in leak-proof \& color-coded or labeled containers,
Contaminated laundry handled as little as possible with minimal agitation" respectively. Whereas the vast majority (91\%) "Held the contaminated laundry bags close to their body while transporting it", while "Linens received by nursing staff" item wasn't done by $2 / 3$ of nurses in both settings; being the responsibility of auxiliary staff. 
Table (3): Subjects distributed according to soiled/contaminated laundry handling (n=46)

\begin{tabular}{|c|c|c|c|c|c|c|c|c|}
\hline \multirow{3}{*}{ Soiled/contaminated laundry handling } & \multirow{3}{*}{ Hospital } & \multicolumn{6}{|c|}{ Done } & \multirow{3}{*}{$\boldsymbol{P}$} \\
\hline & & \multicolumn{2}{|c|}{ Correctly } & \multicolumn{2}{|c|}{ Incorrectly } & \multicolumn{2}{|c|}{ Not done } & \\
\hline & & No. & $\%$ & No. & $\%$ & No. & $\%$ & \\
\hline \multirow{3}{*}{ 1. Soiled linens inspected for any diapers } & $\mathbf{A}$ & 20 & 90.0 & 2 & 9.1 & $\mathbf{0}$ & 0.0 & ${ }^{{ }^{M C}} p=$ \\
\hline & B & 23 & 95.8 & $\mathbf{0}$ & $\mathbf{0 . 0}$ & 1 & 4.2 & 0.226 \\
\hline & Total & 43 & 93.5 & 2 & 4.3 & 1 & 2.2 & \\
\hline \multirow{3}{*}{ 2. Linens received by nursing staff } & A & 6 & 27.3 & 2 & 9.1 & 14 & 63.6 & ${ }^{\mathrm{MC}} p=$ \\
\hline & B & 7 & 29.2 & 0 & 0.0 & 17 & 70.8 & 0.460 \\
\hline & Total & 13 & 28.3 & 2 & 4.3 & 31 & 67.4 & \\
\hline \multirow{3}{*}{$\begin{array}{l}\text { 3. Place wet contaminated laundry in leak-proof,\& } \\
\text { color-coded, or labeled containers }\end{array}$} & A & 17 & 77.3 & 5 & 22.7 & 0 & 0.0 & 0619 \\
\hline & B & 17 & 70.8 & 7 & 29.2 & 0 & 0.0 & 0.019 \\
\hline & Total & 34 & 73.9 & 12 & 26.1 & 0 & 0.0 & \\
\hline \multirow{3}{*}{$\begin{array}{l}\text { 4. Contaminated laundry handled as little as possible } \\
\text { with minimal agitation. }\end{array}$} & A & 18 & 81.8 & 4 & 18.2 & 0 & 0.0 & 0084 \\
\hline & B & 14 & 58.3 & 10 & 41.7 & 0 & 0.0 & 0.084 \\
\hline & Total & 32 & 69.6 & 14 & 30.4 & 0 & 0.0 & \\
\hline \multirow{3}{*}{$\begin{array}{l}\text { 5. Contaminated laundry bags were not held close to } \\
\text { the body when transported. }\end{array}$} & A & 2 & 9.1 & 20 & 90.9 & 0 & 0.0 & $\mathrm{FE}_{p=}=$ \\
\hline & B & 2 & 8.3 & 22 & 91.7 & 0 & 0.0 & 1.000 \\
\hline & Total & 4 & 8.7 & 42 & 91.3 & o & 0.0 & \\
\hline \multirow{3}{*}{ 6. Soiled linen not sorted or rinsed in used location } & A & 22 & $\mathbf{1 0 0 . 0}$ & $\mathbf{0}$ & $\mathbf{0 . 0}$ & $\mathbf{0}$ & $\mathbf{0 . 0}$ & \\
\hline & B & 24 & 100.0 & $\mathbf{0}$ & $\mathbf{0 . 0}$ & $\mathbf{0}$ & $\mathbf{0 . 0}$ & \\
\hline & Total & 46 & 100.0 & $\mathbf{0}$ & $\mathbf{0 . 0}$ & $\mathbf{0}$ & $\mathbf{0 . 0}$ & \\
\hline \multirow{3}{*}{$\begin{array}{l}\text { 7. Contaminated laundry placed\& transported in } \\
\text { red bags or containers labeled as a biohazard }\end{array}$} & $\mathbf{A}$ & 22 & 100.0 & $\mathbf{0}$ & 0.0 & $\mathbf{0}$ & 0.0 & \\
\hline & B & 24 & 100.0 & $\mathbf{0}$ & $\mathbf{0 . 0}$ & 0 & 0.0 & \\
\hline & Total & 46 & 100.0 & 0 & 0.0 & 0 & 0.0 & \\
\hline
\end{tabular}

$M C$ : Monte Carlo for Chi-square test

$F E$ : Fisher Exact for Chi-square test

Figure (1) Revealed that, all subjects did not "Rinse soiled linen in a location of use" correctly, as well $2 / 3$ "Placed and transport the laundry in bags or leak-proof containers labeled with biohazard symbol or put laundry in red bags" correctly. Likewise, $2 / 3$ "Picked up the linens without sorting" incorrectly. It was also noticed that "Infected laundry not wrapped in yellow plastic bags by nearly $1 / 3$ of subjects.

Figure (1): Subjects distribution according to isolated or infected laundry handling (n=46)

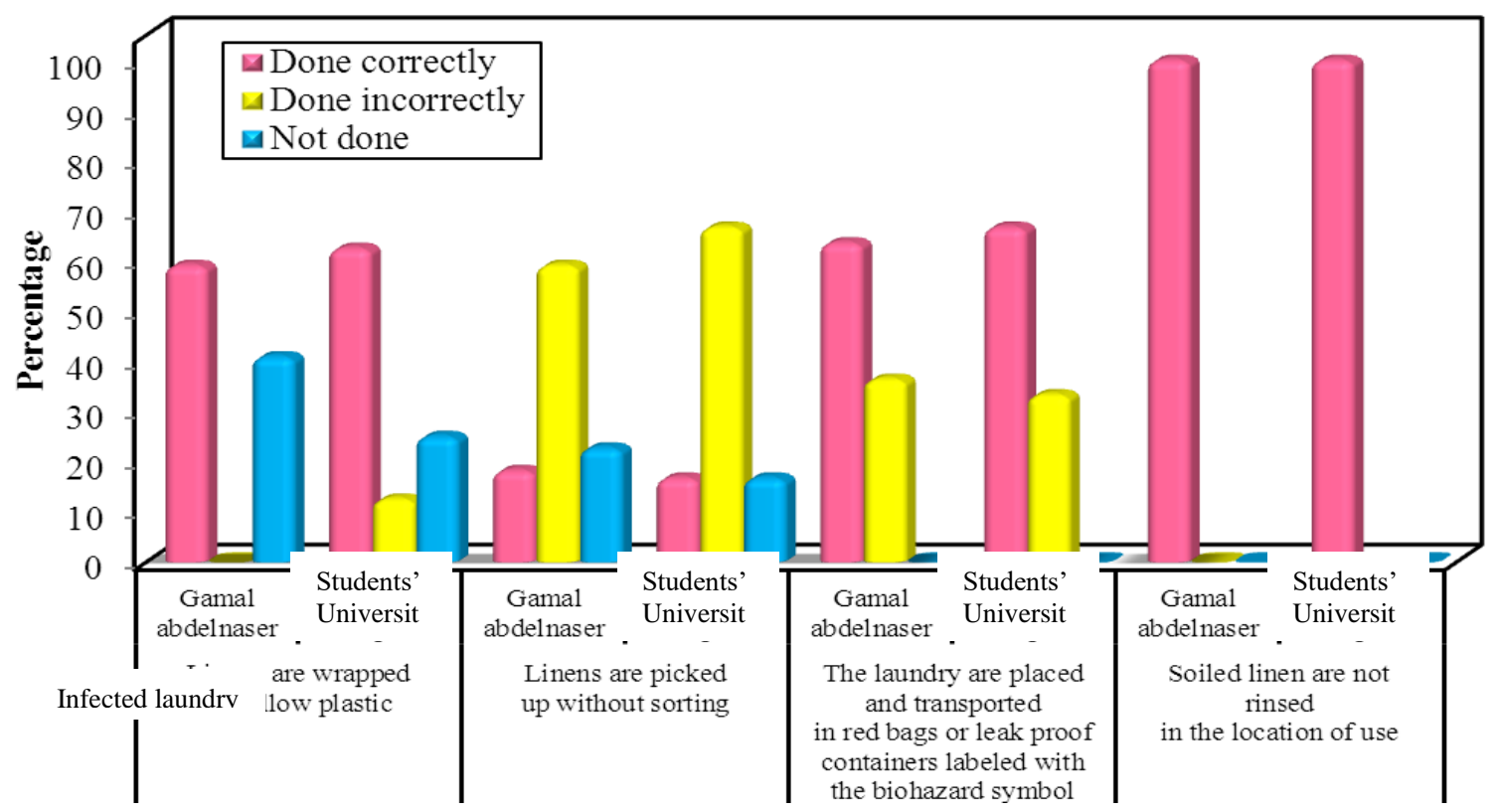


Figure (2) illustrates that; a majority of staff performed two items correctly, namely; "Discard the disposable soiled laundry into puncture-resistant, leak-proof, and labeled red bags with a biohazard symbol", and" Inspected soiled laundry prior processing", representing (100\% \& 83.5\%) respectively. On the other hand, it was noticed that $1 / 4$ of the overall staff still incorrectly "Laundering their uniform at the hospital". Additionally, nearby $2 / 3$ for both groups don't correctly "Inspect linens if intact or not", and "Neglected their hand hygiene after used soiled linen handling".

Figure (2): Subjects distribution according to laundry handling with infection control practices

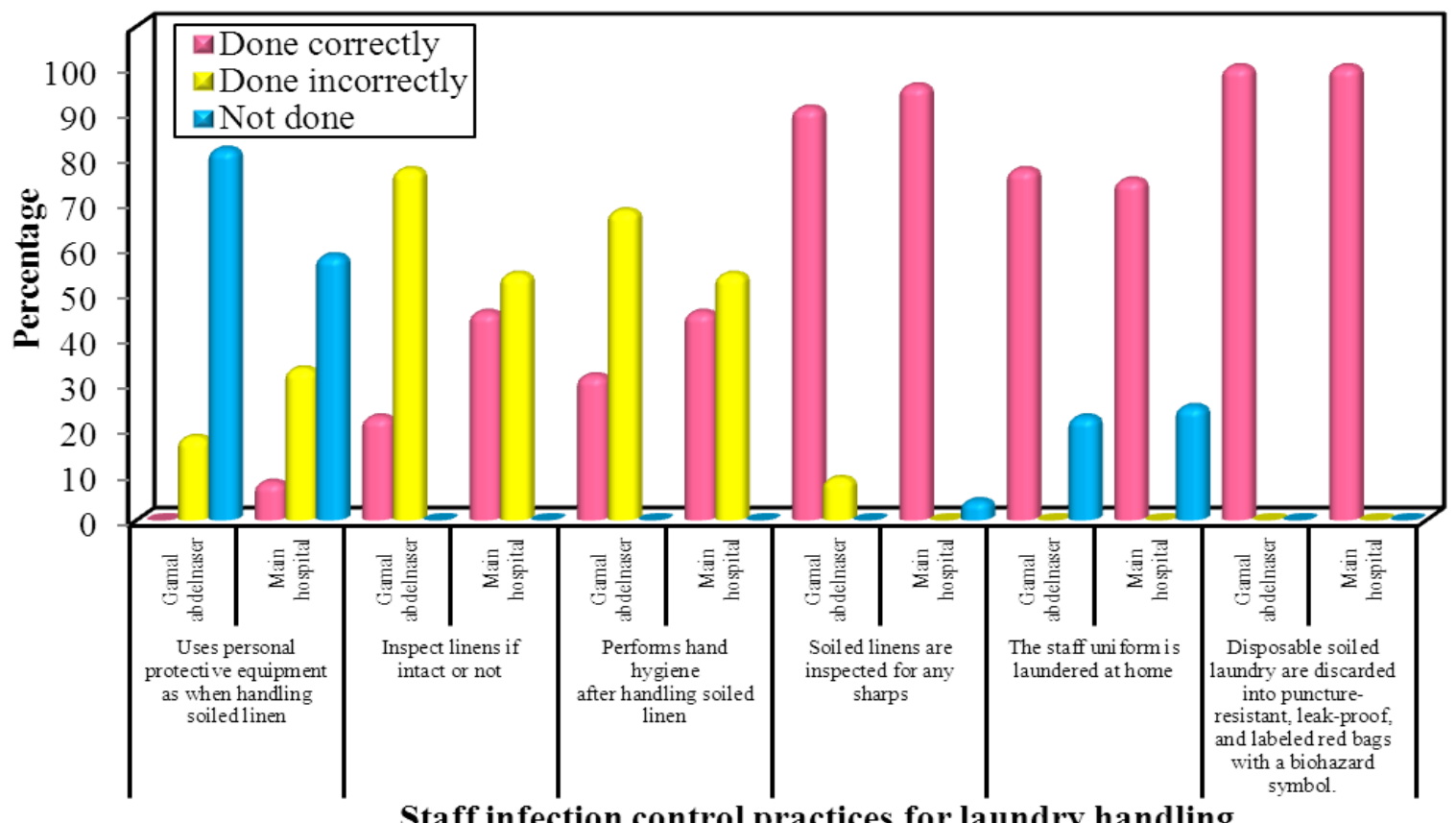

Table (4) shows that; "Laundry management, Adequacy of hot water and detergent solution, as well as Laundry management according to manufacturer instructions for practices" performed by all laundry management staff in both settings (100\%). While, the sub-items "Use multiple laundry bags/carts, Ensure that bags not more than twothirds full, keeping loads at hip height, and Use reaching aids to pulling the linen forward" were not performed by both settings (100\%). Nearly half of the subjects are not aware of specific workplace practices representing $47.8 \%$. However, a significant correlation was detected between the availability of PPE at all times and laundry processing management $(\mathrm{r}=0.029$ at $\boldsymbol{p} \leq 0.05)$.

Table (4): Subjects distribution according to laundry processing management practices.

\begin{tabular}{|c|c|c|c|c|c|c|c|c|}
\hline \multirow[b]{2}{*}{ Laundry management practices } & \multirow{2}{*}{ Hospital } & \multicolumn{2}{|c|}{ Yes } & \multicolumn{2}{|c|}{ Somehow } & \multicolumn{2}{|c|}{ No } & \multirow{2}{*}{$\mathbf{P}$} \\
\hline & & No. & $\%$ & No. & $\%$ & No. & $\%$ & \\
\hline 1- Performed laundry management & $\begin{array}{c}\text { A } \\
\text { B } \\
\text { Total } \\
\end{array}$ & $\begin{array}{l}22 \\
24 \\
46\end{array}$ & $\begin{array}{l}100.0 \\
100.0 \\
100.0\end{array}$ & $\begin{array}{l}\text { 0 } \\
\text { 0 } \\
\text { 0 }\end{array}$ & $\begin{array}{l}\text { 0.0 } \\
\mathbf{0 . 0} \\
\mathbf{0 . 0}\end{array}$ & $\begin{array}{l}\mathbf{0} \\
\mathbf{0} \\
\mathbf{0}\end{array}$ & $\begin{array}{l}0.0 \\
0.0 \\
\mathbf{0 . 0}\end{array}$ & - \\
\hline 2- Aware of specific workplace practices & $\begin{array}{c}\text { A } \\
\text { B } \\
\text { Total }\end{array}$ & $\begin{array}{c}9 \\
15 \\
24\end{array}$ & $\begin{array}{l}40.9 \\
62.5 \\
52.2\end{array}$ & $\begin{array}{c}13 \\
9 \\
22\end{array}$ & $\begin{array}{l}59.1 \\
37.5 \\
47.8\end{array}$ & $\begin{array}{l}\text { 0 } \\
\text { 0 } \\
\text { 0 }\end{array}$ & $\begin{array}{l}\mathbf{0 . 0} \\
\mathbf{0 . 0} \\
\mathbf{0 . 0}\end{array}$ & 0.143 \\
\hline $\begin{array}{l}\text { 3- Eliminate the need to lift, carry or handle heavy bags of } \\
\text { soiled laundry by } \\
\text { a-Using mechanical aids such as carts and slings }\end{array}$ & $\begin{array}{c}\text { A } \\
\text { B } \\
\text { Total }\end{array}$ & $\begin{array}{l}0 \\
0 \\
0\end{array}$ & $\begin{array}{l}0.0 \\
0.0 \\
0.0\end{array}$ & $\begin{array}{l}8 \\
11 \\
19\end{array}$ & $\begin{array}{l}36.4 \\
45.8 \\
41.3\end{array}$ & $\begin{array}{l}14 \\
13 \\
27\end{array}$ & $\begin{array}{l}63.6 \\
54.2 \\
58.7\end{array}$ & \\
\hline
\end{tabular}




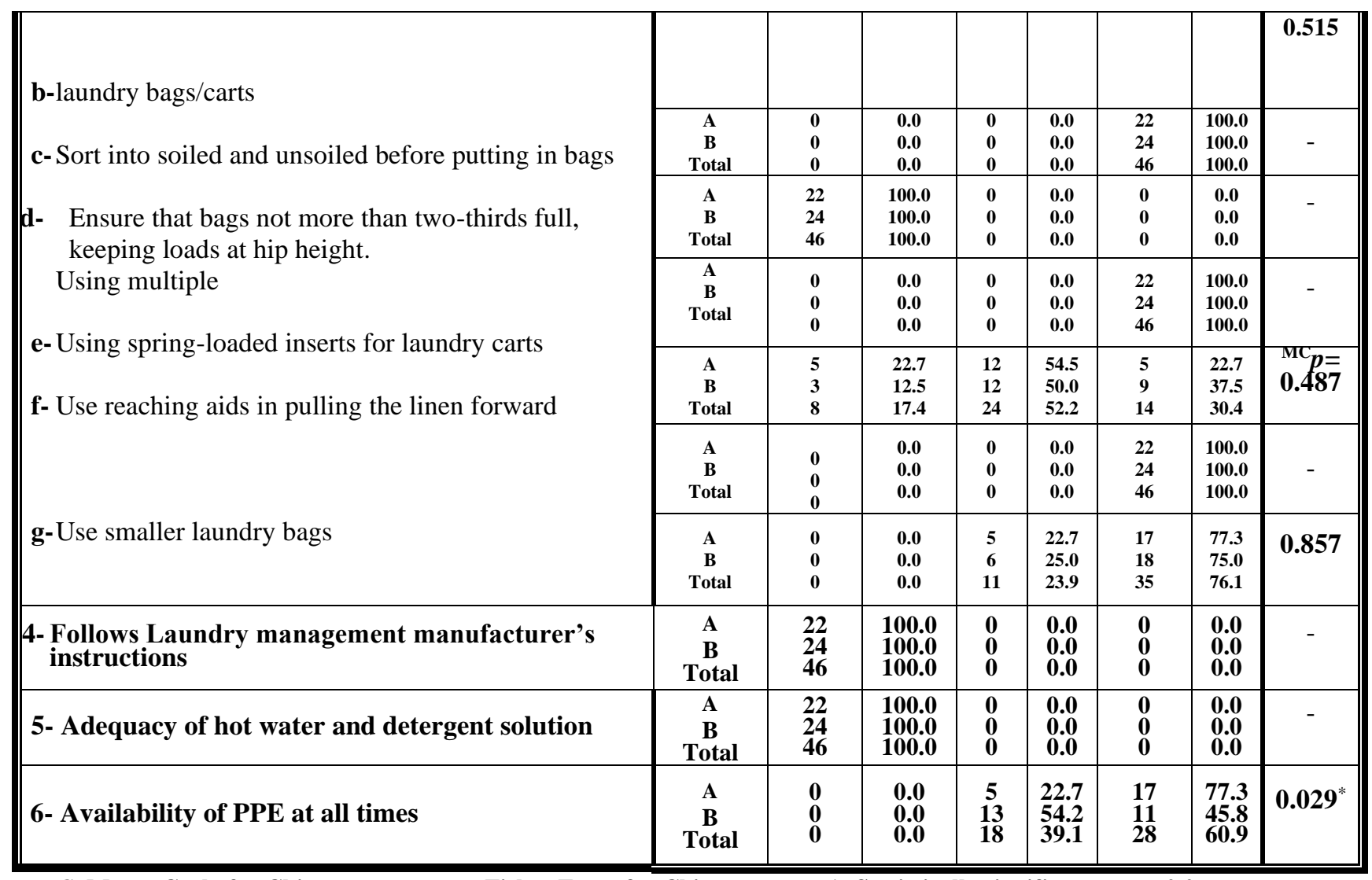

$M C$ : Monte Carlo for Chi-square test, FE: Fisher Exact for Chi-square test, *: Statistically significant at $p \leq 0.05$

Table (5) reveals that the overall staff laundry handling practices score was satisfactory in both settings, with no significant correlations between both settings based on their laundry handling practices.

Table (5): Subjects distribution according to laundry handling practice

\begin{tabular}{|c|c|c|c|c|c|c|c|}
\hline \multirow{2}{*}{ Variables } & \multicolumn{2}{|c|}{ Total $(n=46)$} & \multicolumn{2}{|c|}{$A(n=22)$} & \multicolumn{2}{|c|}{$B(n=24)$} & \multirow{2}{*}{${ }^{F E} p$} \\
\hline & No. & $\%$ & No. & $\%$ & No. & $\%$ & \\
\hline $\begin{array}{l}\text { Clean laundry handling } \\
<60 \text { unsatisfactory } \\
>60 \text { satisfactory }\end{array}$ & $\begin{array}{c}0 \\
46\end{array}$ & $\begin{array}{c}0.0 \\
100.0\end{array}$ & $\begin{array}{c}0 \\
22\end{array}$ & $\begin{array}{c}0.0 \\
100.0\end{array}$ & $\begin{array}{c}0 \\
24\end{array}$ & $\begin{array}{c}0.0 \\
100.0\end{array}$ & - \\
\hline $\begin{array}{l}\text { Soiled / contaminated laundry } \\
\text { handling } \\
\quad<60 \text { unsatisfactory } \\
\quad>60 \text { satisfactory } \\
\end{array}$ & $\begin{array}{c}2 \\
44\end{array}$ & $\begin{array}{c}4.3 \\
95.7\end{array}$ & $\begin{array}{c}2 \\
20\end{array}$ & $\begin{array}{c}9.1 \\
90.9\end{array}$ & $\begin{array}{c}0 \\
24\end{array}$ & $\begin{array}{c}0.0 \\
100.0\end{array}$ & 0.223 \\
\hline $\begin{array}{l}\text { Isolated or Infectious laundry } \\
\text { handling } \\
\quad<60 \text { unsatisfactory } \\
>60 \text { satisfactory } \\
\end{array}$ & $\begin{array}{c}4 \\
42\end{array}$ & $\begin{array}{c}8.7 \\
91.3\end{array}$ & $\begin{array}{c}2 \\
20\end{array}$ & $\begin{array}{c}9.1 \\
90.9\end{array}$ & $\begin{array}{c}2 \\
22\end{array}$ & $\begin{array}{c}8.3 \\
91.7\end{array}$ & 1.000 \\
\hline $\begin{array}{l}\text { infection control staff practices } \\
\text { responsible for laundry handling } \\
<60 \text { unsatisfactory } \\
\quad>60 \text { satisfactory }\end{array}$ & $\begin{array}{c}3 \\
43\end{array}$ & $\begin{array}{c}6.5 \\
93.5\end{array}$ & $\begin{array}{c}2 \\
20\end{array}$ & $\begin{array}{c}9.1 \\
90.9\end{array}$ & $\begin{array}{c}1 \\
23\end{array}$ & $\begin{array}{c}4.2 \\
95.8\end{array}$ & 0.600 \\
\hline $\begin{array}{l}\text { laundry Processing practices } \\
<60 \text { unsatisfactory } \\
>60 \text { satisfactory }\end{array}$ & $\begin{array}{c}46 \\
0\end{array}$ & $\begin{array}{c}100.0 \\
0.0\end{array}$ & $\begin{array}{c}22 \\
0\end{array}$ & $\begin{array}{c}100.0 \\
0.0\end{array}$ & $\begin{array}{c}24 \\
0\end{array}$ & $\begin{array}{c}100.0 \\
0.0\end{array}$ & - \\
\hline $\begin{array}{l}\frac{\text { Overall }}{<60 \text { unsatisfactory }} \\
\quad>60 \text { satisfactory }\end{array}$ & $\begin{array}{c}3 \\
43\end{array}$ & $\begin{array}{c}6.5 \\
93.5\end{array}$ & $\begin{array}{c}2 \\
20\end{array}$ & $\begin{array}{c}9.1 \\
90.9\end{array}$ & $\begin{array}{c}1 \\
23\end{array}$ & $\begin{array}{c}4.2 \\
95.8\end{array}$ & 0.600 \\
\hline
\end{tabular}


Table (6) demonstrates no significant relations between overall staff laundry processing safety practical levels and their socio-demographic data except for gender, where $(\mathrm{FEp}=0.003)$

Table (6): -Relation between overall laundry processing safety practice levels and sociodemographic data.

\begin{tabular}{|c|c|c|c|c|c|c|c|c|c|c|c|c|c|}
\hline \multirow[t]{3}{*}{ Variables } & \multicolumn{4}{|c|}{$\begin{array}{c}\text { A } \\
\text { Overall } \\
\text { practice }(\mathbf{n}=22)\end{array}$} & \multicolumn{4}{|c|}{$\begin{array}{c}\text { B } \\
\text { Overall } \\
\text { practice }(n=24)\end{array}$} & \multicolumn{4}{|c|}{\begin{tabular}{|c|} 
Total sample \\
Overall \\
practice $(n=46)$ \\
\end{tabular}} & \multirow{3}{*}{$p$} \\
\hline & \multicolumn{2}{|c|}{$\begin{array}{c}<60 \text { Poor } \\
(n=2)\end{array}$} & \multicolumn{2}{|c|}{$\begin{array}{c}\geq 60 \text { Good } \\
(n=20)\end{array}$} & \multicolumn{2}{|c|}{$\begin{array}{c}<60 \text { Poor } \\
(n=1)\end{array}$} & \multicolumn{2}{|c|}{$\begin{array}{c}\geq 60 \text { Good } \\
(n=23)\end{array}$} & \multicolumn{2}{|c|}{$\begin{array}{c}<60 \text { Poor } \\
(n=3)\end{array}$} & \multicolumn{2}{|c|}{$\begin{array}{c}\geq 60 \mathrm{Good} \\
(\mathrm{n}=43)\end{array}$} & \\
\hline & No. & $\%$ & No. & $\%$ & & $\%$ & No. & $\%$ & No. & $\%$ & No & $\%$ & \\
\hline $\begin{array}{l}\text { Gender } \\
\bullet \text { Male } \\
\text { - Female }\end{array}$ & $\begin{array}{l}1 \\
1\end{array}$ & $\begin{array}{l}50.0 \\
50.0\end{array}$ & $\begin{array}{c}\mathbf{0} \\
20\end{array}$ & $\begin{array}{c}0.0 \\
100.0\end{array}$ & $\begin{array}{l}1 \\
0\end{array}$ & $\begin{array}{c}100.0 \\
0.0\end{array}$ & $\begin{array}{c}0 \\
23\end{array}$ & $\begin{array}{c}0.0 \\
100.0\end{array}$ & $\begin{array}{l}2 \\
1\end{array}$ & $\begin{array}{l}66.7 \\
33.3\end{array}$ & $\begin{array}{c}0 \\
43\end{array}$ & $\begin{array}{c}0.0 \\
100.0\end{array}$ & $\begin{array}{c}{ }^{\mathrm{FE}} p= \\
\mathrm{O.003}^{*}\end{array}$ \\
\hline $\begin{array}{l}\text { Age } \\
\quad \cdot 20-29 \\
\quad \cdot 30-39 \\
\quad \cdot 40-49 \\
\cdot 50-59 \text { years }\end{array}$ & $\begin{array}{l}\mathbf{0} \\
\mathbf{1} \\
\mathbf{1} \\
\mathbf{0}\end{array}$ & $\begin{array}{c}0.0 \\
50.0 \\
50.0 \\
0.0\end{array}$ & $\begin{array}{l}6 \\
9 \\
2 \\
3\end{array}$ & $\begin{array}{l}30.0 \\
45.0 \\
10.0 \\
15.0\end{array}$ & $\begin{array}{l}\mathbf{0} \\
\mathbf{0} \\
\mathbf{1} \\
\mathbf{0}\end{array}$ & $\begin{array}{c}\mathbf{0 . 0} \\
0.0 \\
100.0 \\
0.0\end{array}$ & $\begin{array}{c}4 \\
10 \\
6 \\
3\end{array}$ & $\begin{array}{l}17.4 \\
43.5 \\
26.1 \\
13.0\end{array}$ & $\begin{array}{l}\mathbf{0} \\
1 \\
2 \\
\mathbf{0}\end{array}$ & \begin{tabular}{c|}
0.0 \\
33.3 \\
66.7 \\
0.0
\end{tabular} & $\begin{array}{l}10 \\
19 \\
8 \\
6\end{array}$ & $\begin{array}{l}23.3 \\
44.2 \\
18.6 \\
14.0\end{array}$ & $\begin{array}{l}{ }^{M C} \mathrm{p}= \\
0.314\end{array}$ \\
\hline $\begin{array}{l}\text { Years of experience } \\
\cdot \geq 10 \text { years } \\
\cdot 10 \geq 20 \text { years } \\
\cdot 20 \geq 30 \text { years } \\
\cdot \text { More than } 30 \text { years }\end{array}$ & $\begin{array}{l}\mathbf{0} \\
\mathbf{1} \\
\mathbf{1} \\
\mathbf{0}\end{array}$ & $\begin{array}{c}\mathbf{0 . 0} \\
\mathbf{5 0 . 0} \\
\mathbf{5 0 . 0} \\
\mathbf{0 . 0}\end{array}$ & $\begin{array}{l}6 \\
7 \\
4 \\
3\end{array}$ & $\begin{array}{l}30.0 \\
35.0 \\
20.0 \\
15.0\end{array}$ & $\begin{array}{l}\mathbf{0} \\
\mathbf{0} \\
\mathbf{1} \\
\mathbf{0}\end{array}$ & $\begin{array}{c}0.0 \\
0.0 \\
100.0 \\
0.0\end{array}$ & $\begin{array}{l}4 \\
9 \\
6 \\
4\end{array}$ & $\begin{array}{l}17.4 \\
39.1 \\
26.1 \\
17.4\end{array}$ & $\begin{array}{l}\mathbf{0} \\
1 \\
2 \\
\mathbf{0}\end{array}$ & \begin{tabular}{c|}
0.0 \\
33.3 \\
66.7 \\
0.0
\end{tabular} & $\begin{array}{l}10 \\
16 \\
10 \\
7\end{array}$ & $\begin{array}{l}23.3 \\
37.2 \\
23.3 \\
16.3\end{array}$ & $\begin{array}{l}{ }^{M C} p= \\
0.500\end{array}$ \\
\hline \begin{tabular}{|l} 
Position \\
- Nursing aides staff \\
- Junior newly \\
graduate) \\
- Senior staff \\
$\quad$ (diploma) \\
- Supervisor nurse \\
- Head-nurse
\end{tabular} & $\begin{array}{l}1 \\
1 \\
0\end{array}$ & $\begin{array}{c}50.0 \\
50.0 \\
0.0 \\
\\
0.0 \\
0.0\end{array}$ & $\begin{array}{l}3 \\
1\end{array}$ & $\begin{array}{c}10.0 \\
45.0 \\
25.0 \\
15.0 \\
5.0\end{array}$ & $\begin{array}{l}\mathbf{0} \\
\mathbf{0}\end{array}$ & \begin{tabular}{r|}
100.0 \\
0.0 \\
0.0 \\
0.0 \\
0.0
\end{tabular} & $\begin{array}{l}2 \\
10\end{array}$ & \begin{tabular}{c|}
8.7 \\
43.5 \\
21.7 \\
21.7 \\
4.3
\end{tabular} & 2 & $\begin{array}{r}66.7 \\
33.3 \\
0.0 \\
0.0 \\
0.0\end{array}$ & $\begin{array}{l}8 \\
2\end{array}$ & $\begin{array}{r}9.3 \\
44.2 \\
23.3 \\
18.6 \\
4.7\end{array}$ & $\begin{array}{l}{ }^{M C} \mathrm{p}= \\
0.157\end{array}$ \\
\hline $\begin{array}{l}\text { Education level } \\
\text { - Diploma } \\
\text { - Bachelor degree } \\
\text { - Others }\end{array}$ & $\begin{array}{l}1 \\
\mathbf{0} \\
\mathbf{1}\end{array}$ & $\begin{array}{c}\mathbf{5 0 . 0} \\
\mathbf{0 . 0} \\
\mathbf{5 0 . 0}\end{array}$ & $\begin{array}{c}12 \\
6 \\
2\end{array}$ & $\begin{array}{l}\text { 60.0 } \\
30.0 \\
10.0\end{array} \mid$ & $\begin{array}{l}\mathbf{0} \\
\mathbf{0} \\
\mathbf{1}\end{array}$ & $\begin{array}{c}0.0 \\
0.0 \\
100.0\end{array} \mid$ & $\begin{array}{c}14 \\
7 \\
2\end{array}$ & $\begin{array}{c}60.9 \\
30.4 \\
8.7\end{array}$ & $\begin{array}{l}1 \\
\mathbf{0} \\
\mathbf{2}\end{array}$ & $\begin{array}{c}33.3 \\
0.0 \\
66.7\end{array}$ & $\begin{array}{c}26 \\
13 \\
4\end{array}$ & $\begin{array}{c}60.5 \\
30.2 \\
9.3\end{array}$ & $\begin{array}{l}{ }^{M C} p= \\
0.063\end{array}$ \\
\hline \begin{tabular}{|l} 
Workshop \\
$\bullet$ Yes \\
$\bullet$ No \\
\end{tabular} & $\begin{array}{l}\mathbf{0} \\
2\end{array}$ & $\left|\begin{array}{c}0.0 \\
100.0\end{array}\right|$ & $\begin{array}{c}2 \\
18\end{array}$ & $\begin{array}{l}10.0 \\
90.0\end{array}$ & $\begin{array}{l}\mathbf{0} \\
\mathbf{1}\end{array}$ & $\begin{array}{c}0.0 \\
100.0\end{array}$ & $\begin{array}{c}4 \\
19\end{array}$ & $\begin{array}{l}17.4 \\
82.6\end{array}$ & $\begin{array}{l}\mathbf{0} \\
\mathbf{3}\end{array}$ & $\begin{array}{c}0.0 \\
100.0\end{array}$ & $\begin{array}{c}6 \\
37\end{array}$ & $\begin{array}{l}14.0 \\
86.0\end{array}$ & $\begin{array}{c}{ }^{F E} p= \\
1.000\end{array}$ \\
\hline \begin{tabular}{|l} 
laundry handling staff \\
$\begin{array}{c}\bullet \text { Both nurses and } \\
\text { nurse aides }\end{array}$
\end{tabular} & 2 & 100.0 & 20 & 100.0 & 1 & 100.0 & 23 & 100.0 & 3 & 100.0 & 43 & 100.0 & - \\
\hline
\end{tabular}

$\chi^{2}, p: \chi^{2}$ and $p$ values for Chi-square test

MC: Monte Carlo for Chi-square test

FE: Fisher Exact for Chi-square test 


\section{Discussion}

Safe infection control measures are the cornerstone of health team member care safe practices; thus assessing hemodialysis staff performance is mandatory for improving and enhancing patients' QOL. Most of the present study staff was female. This is attributed to the that Alexandria's Nursing Faculty graduates mainly female nurses with recent male enrolment. Also, it was noted that; almost half of the hemodialysis staff in both settings were junior diploma graduate nurses because the more experienced staff and the bachelor graduates are usually assigned for administrative and intensive care units (ICU) positions.

Furthermore, the majority of study subjects $(87 \%)$ from both settings didn't attend any training or workshops regarding laundry safety; so attention to this serious issue should not be neglected. This is lined with Asmara et al, 2019; who mentioned that nurses and all hospital staff have to be acquainted with the infectious as well as hazardous sources; and proper methods for handling them ${ }^{(1)}$. This knowledge and proper practice go a long toward its safe disposal for healthcare personnel, patients, and community protection; to maintain a green hospital environment. Also, proactively examining these issues reduce the negative impact of poor practices on the hospital environment.

The current results also revealed that hemodialysis staff in both setting's experience ranged from 10 to $\geq 20$ years; which does in line with Shahdadi H, and Rahnama M., 2018, who stated that, the majority of hemodialysis staff experience should not be less than 4 years ${ }^{(12)}$.

On the other hand, both all nurses and nursing aids $(100 \%)$ reported that; laundry handling is their responsibility; this could attribute to increasing their time spent in the hemodialysis unit than other health team members and the frequent need for laundry management in the unit as well as the unclear policy regarding laundry responsibility. Otherwise, these laundry processing practices are advocating for increased environmental consciousness for hemodialysis staff. This is agreed with El-Sayed et al., 2012 and Parida, 2019 who mentioned that; nurses are the most responsible personnel for handling and disposal of all environmental surfaces and they are liable to direct exposure to all types of infectious hazards $(13,14)$. This is contradicted with Mercy hospital report 2019, that all employed staff, specialists; allied health professionals are supported to meet laundry policy requirements to ensure the appropriate processing and handling of soiled and contaminated linen to minimize infection risk throughout the hospital ${ }^{(15)}$. Thus; education and research opportunities should be available for them to enhance the development of hospital initiatives for fostering environmentally friendly policies and programs.

Concerning clean laundry handling assessment, it was noticed that, items such as "Counted and sorted then picked up linens and Inspection if intact" were incorrectly done by nearly half of the staff in both settings. Furthermore, "Linens were inspected in the middle of hemodialysis unit at a low grounded level" this may touch the floor, as well most of the torn or stained linens were noticed to be re-used for patients instead of discarding; this approach is considered as a source of hazard and infection. This is contradicting with Mclay report in 2013 that; clean laundry is defined as "Any linen that, has not been used since it was last laundered". (16) Otherwise, continuous monitoring for the presence of regulated medical waste (RMW) such as infectious materials, sharps, and medications wastes, that are considered environmentally hazardous in the laundry, instead of being discarded into special containers; should be emphasized to maintain patient's quality of care and safety in the workplace. 
Moreover, McLay stressed that; storing linen within the hospital should be in a designated clean linen room and has to be covered to protect it from dust and dirt until use ${ }^{(16)}$. Also, laundry responsible staff should ensure that linen is on clean shelves at a minimum of 8 inches away from the floor, and 2 inches away from walls, to allow easy cleaning access. Similarly, ensure that the clean laundry room door remains usually closed with restricted access ${ }^{(2)}$. On the same line, Mercy hospital's laundry policy mentioned that; clean linen must be stored in a clean, dry, dust-free area (portable covered carts, or a closet or room) that is geographically separated from soiled linen to prevent its contamination ${ }^{(15)}$.

However, items of clean laundry handling assessment were not done by the study subjects, which can be rationalized by that; assigned staff work have work overload with poor training regarding infection control experiences. Shahdadi H. and Rahnama M, 2018 stressed that; "Well trained hospital staff enhances crisis management and hazard prevention". (12) In spite, McLay mentioned that "Understanding the patients' IC requirement is necessary to prevent the spread of infection, thus; it is important to check linen cleanliness being an integral part of healthcare activity". Furthermore, to ensure that linens are transported and stored appropriately to avoid infection hazards and cost burden ${ }^{(16)}$. Thus; hospital staff development is an initiative that fosters hospitals' environmentally friendly policies and programs.

Along with the current results; practices regarding "Linens that are still dirty or not washed properly are collected and returned to the laundry to undergo rewashing procedure" were not done in both settings groups. This could be explained by hemodialysis nurses as; they didn't attend any training, workshops, or educational training related to safe laundry processing; in addition to, lacking linen supply and limited time with the increased hemodialysis patients' turnover.
Likewise, CDC 2018 declared that; emphasized the purpose of checking laundered hospital textiles to ensure their cleanliness and safety for patients and staff with uninterrupted implementation of healthcare activities. Most hemodialysis staff assumes that; the returned laundry is clean; therefore announcements regarding safely checking laundry for cleanliness and appropriateness are mandatory to improve safe laundry processing, together with preventing infection (17).

Also, current results illustrated that; most of the "Soiled /contaminated laundry handling" items were done correctly by almost all staff, it could be due to restricting quality assurance monitoring and supervision in both settings. As well, it was noticed that; the vast majority of subjects "Held the contaminated laundry bags close to their body while transporting it". This is contradicted with OSHA recommendation; which defined contaminated laundry as "Laundry which is soiled with blood or other potentially infectious materials or may contain sharps". However, laundry occupational hazards are numerous, so contaminated laundry bags should not be held close to the body or squeezed during transportation to avoid punctures from improperly discarded syringes or sharp objects ${ }^{(10,18,19)}$. As well used laundry should not be shaken and held away from the body to avoid staff clothing contamination (20).

On the other hand, the item: "Linens are received by nursing staff" was not done by $2 / 3$ of the nursing staff in both settings. This agrees with the statement of "Nurses are responsible to oversee the cleaning of linens and monitor its protection from contamination for the avoidance of infection". However, laundry departments are assigned to various roles within linen services, so the laundry services team should be mandatory working as working staff, while nurses act as a supervisor or team leader oversees the laundry teamwork services for assistants. ${ }^{(15,20)}$ This helps in developing or reviewing and amending trusted procedural documentation. 
Also $2 / 3$ of subjects "Picked up linens without sorting" incorrectly, as they used to hold laundries close to their clothes or either creep or slid laundries on departments' floor. It was also observed that; if sorting was performed, the assigned staff wasn't wearing any PPE. This is somehow linked with healthcare facilities' policies; that emphasize that linen collection, transportation, and storage should be handled carefully to avoid dispersal of microorganisms into the environment and contact with staff clothing; using appropriate PPE during soiled linen handling for prevention of exposure of skin and mucous membrane to blood and body substances; Additionally, the used linen must be "bagged" at the location of its use in an appropriate laundry receptacle ${ }^{(13,20)}$.

On the other hand, "Isolated or infected laundry handling were not wrapped in yellow plastic bags" by $1 / 3$ of subjects. As well, a majority of staff "Inspected soiled laundry before processing and discarded disposable soiled laundry into puncture-resistant, leak-proof, and labeled red bags with a biohazard symbol" correctly. However, OSHA confirmed that an outline of bloodborne standards for handling the contaminated laundry procedures such as: handling contaminated laundry as little as possible with minimal agitation, using bag contaminated laundry at its location of use, don't sort or rinsing laundry at the hemodialysis unit where it was used, place wet contaminated laundry in leak-proof, and color-coded or labeled containers, at the location where it was used ${ }^{(17.10)}$. However, contaminated laundry is usually wet with a reasonable likelihood of leakage from the bag or container, thus laundry should be transported in water-proof biohazard symbol labeling or colorcoding bags or containers to the exterior ${ }^{(18,19)}$.

Otherwise, it was detected that; $1 / 4$ of the overall staff were incorrectly "Laundering their uniform at the hospital". On the same line; few hospitals launder staff uniforms. While the staff is expected to use their domestic machines to reduce viable counts of Staphylococcus aureus to below detectable levels at low temperature $\left(40{ }^{\circ} \mathrm{C}\right)$ programs. Thus, domestic uniform laundering is an acceptable alternative to hospital laundering if combined with tumble drying or ironing ${ }^{(21)}$. Study results illustrated that a microbiological sampling of nurses' uniforms was taken before and after a span of duty; a Staphylococcus aureus, Clostridium difficile, and Vancomycin-resistant enterococci were detected on staff uniforms ${ }^{(22,23)}$. Otherwise, it was observed that more than half of the laundry assigned hemodialysis team didn't perform "Hand hygiene after handling of used soiled linen", and their rationale attributed to decreased staff number compared to patients during shifts' time. On contrary; it is strongly recommended by WHO, CDC, Morbidity, and Mortality Weekly Report (MMWR), and OSHA Prevention Guidelines to "Perform hand hygiene immediately after direct inanimate objects contact or with routine patients' activate i.e., touching surfaces likely to be contaminated". (24) However, emphasis on laundry infection transmission prevention by Universal Precautions; hand hygiene (i.e., hand washing or disinfectant hand rub), glove use, and disinfection of environmental surfaces (e.g., bed rails, laundries, department supplies) are strongly needed to be implemented ${ }^{(25)}$. As regards the laundry processing area; laundry sorting practices were performed in both settings by all staff (100\%). This is lined with Parida 2019 who stated that; the staff is responsible to ensure that soiled and contaminated linen is stored, transported, collected as well as appropriately handled, and processing in a manner that minimizes infection risk for patients, staff, and visitors throughout the hospital ${ }^{(14)}$. Likewise damaged linen should be sorted and separated then bagged with a note for repair or review for a replacement to laundry staff (e.g. gowns, fitted sheets, blankets) ${ }^{(23)}$. Similarly, additional measures to prevent environmental contamination through safe handling and sorting of contaminated laundry, supplies, equipment, blood samples, or biohazard containers in areas of hemodialysis ${ }^{(25)}$. 
Regarding "Adequacy of hot water and detergent as well as laundry management practices according to manufacturer instructions in processing area", it was performed from both settings by $(100 \%)$ of all laundry processing staff. As evidenced, a study was conducted to investigate the bacterial killing action of hot and cold wash formulas, according to manufacturer instructions, in heavily soiled linen washing. It is well known that; routinely hospital laundries use formulated chemicals at hightemperature wash waters of $66^{\circ} \mathrm{C}$, at the beginning of the washing process. It was detected that; the most commonly gram-negative rods found were Klebsiella, Enterobacter, and Serratia species, while Staphylococci were the predominant gram-positives. Both cold and hot water washing accompanied with bleach cycle; reduced bacterial counts in fabric. Similarly, the reduction was affected by a $93.3^{\circ} \mathrm{C}$ drying cycle. Coldwater formulas at $31.1^{\circ} \mathrm{C}$ offer an alternative method to reduce energy consumption and maintain bacteriological and esthetic linen quality ${ }^{(26)}$.

However, routine laundry procedures, detergents, and laundry additives help to make clothes, towels, and linens safe to wear or touch. Following the manufacturer's instructions regarding clothing, soap or detergent, and laundry water temperature is mandatory otherwise damage to the clothing or a decrease in the detergent efficacy could occur. ${ }^{(27)}$

Otherwise, practices such as "Use of multiple laundry bags/carts, keeping loads at hip height, use reaching aids to pull the linen forward" were not performed by all subjects. While, references pointed on, a general industry standard for personal fall protection systems to reduce back and workplace injuries. Furthermore, OSHA recommended the use of proper body mechanics along with mechanical assistance through engineering controls, inform of the use of certain equipment as; forklifts and cranes, which are covered under separate regulations with a properly designed workstation to eliminate the exposure to hazards, in addition to employees training regarding safe lifting techniques ${ }^{(17.10,28)}$.

Also, a significant correlation was found between "PPE availability at all times and laundry processing practices" ( $\mathrm{r}=0.029$ at $p \leq 0.05)$. On the same line, $\mathrm{CDC}$ for infection control recommended the use of standard precautions in hemodialysis units including PPE; (gloves, gown, face shields, eyewear, or mask) to prevent contact of $\mathrm{HCW}$ with blood, secretions, excretions, or contaminated items. For example, infection control practices for hemodialysis units restrict the use of common supplies, instruments, medications carts, and medication trays $(25,29)$ Additionally; staff members should wear complete PPE if required to protect themselves from clothing soiling when performing procedures like initiation and termination of dialysis, cleaning of dialyzers and centrifugation of blood. Such protective clothing should be changed if becomes soiled ${ }^{(30,31)}$.

Finally, it can be observed that the overall performed safe laundry practices scores were satisfactory in both settings with no significant relations between overall staff laundry safe practices scores and their socio-demographic characteristics except for gender. This could be explained by the minimal percentage of male nurses working in the dialysis department. Consequently, the weakness of any performed laundry practices processing would expose both staff and patients to hospital infection/contamination hazards.

Thus preventing blood-borne viruses and pathogenic bacteria transmission among chronic hemodialysis patients requires a comprehensive implementation of a specifically designed infection control program; recommended for hemodialysis units, to diminish the opportunities for direct or indirect transmission of infectious agents via contaminated devices, equipment, and supplies, environmental surfaces or even hands of personnel which serve as an intermediate reservoir for pathogens ${ }^{(32,33,34)}$.

\section{Study limitation:}


The current study results cannot be generalized due to sample size limitations. So it must be applied to all health care settings in Alexandria for example.

\section{Conclusion and recommendations}

Based on the present study results, it can be concluded that: a significant correlation between the availability o7. PPE at all safe laundry processing practices, reflecting the satisfactory scores of overall laundry safety practices performed in both settings; with no significant relations between overall staff laundry safety practices scores and their socio-demographic characteristics except for gender.

The recommendation, implementing training prograrss in the dialysis unit regarding the safe practice of handling and disposal of laundry should be organized. Ascertain the multidisciplinary approach to maintain safe laundry handling in all hospital units especially dialysis, which is highly recommended. Periodic monitoring of staff regarding infection control knowledge and practice, as w9. as provide a visible written policy about safety practices handling and disposal of laundry in hemodialysis units.

\section{References}

1. Asmara A., SriHariyati T., Handiyani H., Avia I. Knowledge and performance of nursing students of Kermanshah-Iran regarding the standards of nosocomial infections control: a cross-sectional study. Enfermería Clínica, 29 (2), 36-40, 2019.

2. Centers for Disease Control and Prevention. National Chronic Kidney Disease Fact Sheet. Atlanta (GA): US Department of Health and Human Services, Centers for Disease Control and Prevention; 2017.

3. The Egyptian Society of Nephrology and Transplantation ESNT Hemodialysis Guidelines 2019. J Egypt Soc Nephrol Transplant 2020;20:190.

4. Hill T. Guidelines for the Management of Linen and $\quad$ Laundry. 2012 http://www.eastcheshire.nhs.uk/.

5. Flanagan, E., Cassone, M., Montoya, A., \& Mody, L. Infection Control in Alternative Health Care Settings: An Update. Infectious disease clinics of
North America, 30(3), 785-804. 2016. Doi: 10.1016/j.idc.2016.05.001

6. Pyrek M. Infection Control Today: Standard Precautions When Handling Soiled Linens. PPE \& Standard Precautions, 2012.

Michael K., David N., Dankoff J., Lee K., LaraCrawford E., Roberts M. Clostridium difficile Environmental Contamination within a Clinical Laundry Facility in the USA. FEMS Microbiology Letters, 2016; fnw236 DOI: 10.1093/femsle/fnw236.

Linen handling and laundry policy. Infection Prevention Management Committee. 2013. Available at: http://www.monhealthcare.com/policy/8807/Infect ion-Prevention-and-Control-Committee-

Organization-and-Function

Guidelines for Design and Construction of Health Care Facilities; the Facility Guidelines Institute. 2011. www.haad.ae

10.Omoijiade EN. An assessment of laundry workers' exposure to workplace hazards in secondary and tertiary health facilities in Benin-city, Nigeria. MOJ Public Health. 2018;7(5):252-259. DOI: 10.15406/mojph.2018.07.00239

11.Contaminated Laundry assessment. Occupational Safety \& Health Administration OSHA Hospital. 2012. E-toolhttps://www.osha.gov/SLTC/etools/hospital/laund ry/laundry.html

12. Shahdadi H. and RahnamaM. Experience of Nurses in Hemodialysis Care: A Phenomenological Study. Journal of Clinical Medicine 7(2):30, $2018 . \quad$ DOI: 10.3390/jcm7020030

13.El-Sayed S., Zakaria A. and Gheith N. Intervention Program for Nurses about Health Care Waste Management. Research Journal of Medicine and Medical Sciences, 7(1): 25-37, 2012.

14. Parida, A., Capoor, M. R., \& Bhowmik, K. T. (2019). Knowledge, attitude, and practices of Biomedical Waste Management rules, 2016; Bio- 
medical Waste Management (amendment) rules, 2018; and Solid Waste Rules, 2016, among health-care workers in a tertiary care setup. Journal of laboratory physicians, 11(4), 292-299. https://doi.org/10.4103/JLP.JLP_88_19

15. Mercy hospital; laundry policy, reviewed February 2019. Available at: https://www.mercycq.com/mh/mercy-linen.

16. McLay C. Environment of Care Leader, linen storage. Decision Health. Washington. 2013.

17. CDC Drafts Guideline on Infection Control in Healthcare Personnel. Centers for Disease Control and Prevention. 2018. Available at: https://www.regulations.gov/document?D=CDC2018-0099-0001

18. Analysis of Sustainability in Hospital Laundry: The Social, Environmental, and Economic (Cost) Risks. Lopes C., Scavarda A., Carvalho M., Vaccaro G. and Korzenowski A. Resources, 2019, 8,37

19.Flynn M., Coughlan P., Forde L., Mc Donnell N. Guidelines on Infection Prevention and Control Nursing. 2012, HSE South. Community and Disability Services. Section (9) Laundry.

20.University Hospitals Birmingham NHS Foundation Trust (UHB); Linen and laundry. 2019. available at: https://hgs.uhb.nhs.uk/foi-0136-2019-20-linenand-laundry/

21. Heudorf U, Gasteyer S, Müller M, Serra N, Westphal T, Reinheimer C, Kempf V. 2017. Handling of laundry in nursing homes in Frankfurt am Main, Germany: Laundry and professional clothing as potential pathways of bacterial transfer. GMS. Hygiene and Infection Control 12:20. 2016. DOI 10.3205/dgkh000305.

22. Laird $\mathrm{K}$ et al. Domestic laundering of nurses' uniforms: what are the risks? Nursing Times [online]; 114: 2. 2018.

23.Desta1 E., Gebrie M., and Dachew B. Nurse uniform wearing practices and associated factors among nurses working in Northwest Ethiopia: a cross-sectional institution based study. BMC Nursing. 14: 65. 2015.

24.Pittet D. Hand hygiene: From research to action. Journal of Infection Prevention. 18 (3), 2017.

25. Said S. A. Khamisa, Yasin S. Yasina, Mahmoud M. Omaraa, Nagy E. N. Saleh. Safety measures in Dakhlia hemodialysis units. 31(2). 2018. Faculty of Medicine, Menoufia University.

26. Dirk P. Bockmühl, Jan Schages, Laura Rehberg. Laundry and textile hygiene in healthcare and beyond. Microb Cell. 2019, 6(7): 299306. 2019. DOI: $10.15698 / \mathrm{mic} 2019.07 .682 \mathrm{PMCI}$ D: PMC6600116

27. Methicillin-resistant Staphylococcus aureus (MRSA), Effectiveness of Routine Laundry Procedures. 2015. Available at: file:///D:/paper/clean\%20laundry/paper/net\%20ref erences \%20new/Laundry\%20\%20MRSA\%20 $\% 20 \mathrm{CDC} \% 20$ manufacturer.html

28. Safe lifting techniques: How to lift safely. Indiana University. $2020 . \quad$ Available at: https://protect.iu.edu/environmentalhealth/occupational-health/ergoprogram/back/safelift.html

29. Ahmadpour B, Ghafourifard M, Ghahramanian A. Trust towards nurses who care for hemodialysis patients: a cross-sectional study. Scand J Caring Sci. 34(4):1010-1016. 2020. DOI: $10.1111 /$ scs. 12809 .

30. Gork, I., Gross, I., Cohen, M.J. et al. Accessrelated infections in two haemodialysis units: results of a nine-year intervention and surveillance program. Antimicrob Resist Infect Control. 8, 105, 2019. https://doi.org/10.1186/s13756-0190557-8

31. Cook E. Surveillance for infections in long-term care. National Healthcare Safety Network (NHSN) Training. SPICE: North Carolina, 2017.

32.Bakarman, M. A., Felimban, M. K., Atta, M. M., \& Butt, N. S. (2019). The effect of an educational program on quality of life in patients undergoing hemodialysis in western Saudi Arabia. Saudi 
medical journal, 40(1), 66-71. Assiut Scientific Nursing Journal, 2 - 3, 1 - 8, https://doi.org/10.15537/smj.2019.1.23401 2014.

33. Abd-Alfatah A., Ahmad A., and Mohamed F. 34.Ali S., Mohammad Z., Abdelmohsen S. Awareness Assessment of Nurses' Knowledge and Practice of Nurses Regarding Hemodialysis Related to Nursing Care of Children Undergoing Complications. Assiut Scientific Nursing Hemodialysis at Assiut City. Journal 6(15), $2018 . \quad$ DOI: 